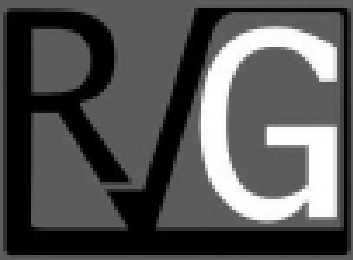

Año 24 No. 88

Octubre - Diciembre 2019

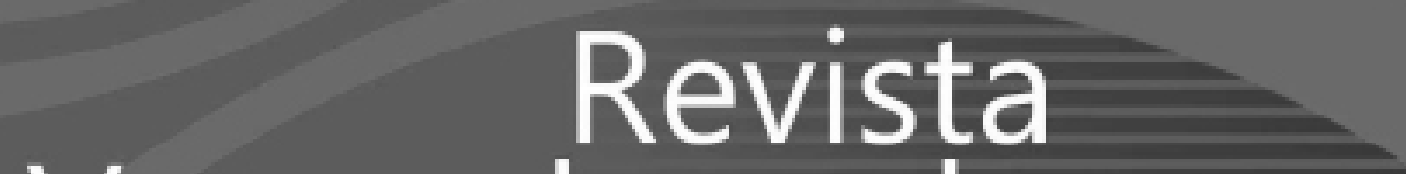

Venezolana de

verencla

UNIVERSIDAD DEL ZULIA (LUZ)

Facultad de Ciencias Económicas y Sociales

Centro de Estudios de la Empresa 


\title{
Sustentabilidad como estrategia competitiva en la gerencia de pequeñas y medianas empresas en México
}

\author{
Ortiz Palafox, Karla Haydee ${ }^{1}$
}

\section{Resumen}

El propósito de esta investigación es analizar la sustentabilidad, como estrategia de competitividad en las pymes mexicanas. Para lograr este objetivo se desarrolló un cuestionario que recogía las principales acciones referentes a la sustentabilidad, a través de un análisis factorial comprobatorio. Por lo tanto, esta validación constituye un aporte para la literatura gerencial, administrativa y en materia medioambiental y confirma que las estrategias se pueden implementar con la reorganización de variables relacionadas con la estructura, los procesos y sistemas de la organización. Se concluye que las pymes del estado de Jalisco son propensas al riesgo de mercado debido a la falta de estrategias para enfrentar los movimientos externos y de competitividad, por la ausencia de una estructura legal, corporativa y de políticas de fomento a través de la sustentabilidad, ocasionando pérdida de competitividad. Así mismo la sustentabilidad debe ser considerada como una capacidad que puede generar una ventaja competitiva sostenible para la pyme.

Palabras clave: sustentabilidad; estrategia competitiva; pymes

$\overline{\text { Recibido: } 30-01-19}$ Aceptado: 14-07-19

Es profesora en el Centro Universitario de Ciencias Económicas Administrativas de la Universidad de Guadalajara, cuenta con doctorado en Ciencias Sociales 2013-2017, del padrón de excelencia Conacyt con certificación competencia Internacional, perteneciente a la red de doctorados del Consejo Latinoamericano de Ciencias Sociales -CLACSO.Email: ortizpalafoxkarla@hotmail.com, ORCID 0000-0003-4836-7074 


\title{
Sustainability as a competitive strategy in the management of small and medium enterprises in Mexico
}

\begin{abstract}
The purpose of this research is to deepen the knowledge of important areas: sustainability and the organizational and administrative phenomenon of the implementation of this, as a strategy in SMEs, to achieve this goal a questionnaire was developed that included the main actions related to the sustainability, through a confirmatory factor analysis. Therefore, this validation constitutes a contribution to the management, administrative and environmental literature and confirms that the strategies can be implemented with the reorganization of variables related to the structure, processes and systems of the organization. It is concluded that SMEs, particularly speaking of the state of Jalisco, are prone to market risk due to the lack of strategies to face external movements and competitiveness, due to the absence of a legal, corporate structure and promotion policies through the sustainability, causing loss of competitiveness. Likewise, sustainability must be considered as a capacity that can generate a sustainable competitive advantage for the SME.
\end{abstract}

Keywords: Sustainability; competitive strategy; SMEs.

\section{Introducción}

El presente trabajo, analiza la problemática de la competitividad en las pymes en términos de sustentabilidad, se buscará determinar, identificar y clasificar la problemática que en las pymes existe dentro del estado de Jalisco y se definirá la sustentabilidad como una medida estratégica. La Administración gerencial eficiente realizada dentro de los procesos sustentables, específicamente dentro de las pymes es una herramienta que proporciona grandes beneficios a cualquier empresa. La necesidad de desarrollar y manejar un adecuado control interno operacional con la finalidad de cumplir con las metas y objetivos que se propone la gerencia proporciona ventajas sobre las otras empresas.

La gestión basada en la responsabilidad social tiene el propósito de buscar el equilibrio apropiado entre el reconocimiento de oportunidades de obtener ganancias y la reducción de las pérdidas. Es parte integral de las buenas prácticas de gestión y también es un elemento esencial para el buen gobierno corporativo.

La capacidad para competir en los mercados de bienes y servicios que imperan en la industria y la región por medio de los mejores métodos de producción y de organización genera ventajas competitivas para el desarrollo y éxito (M. Peris 2017). En México los programas de apoyo para las pymes cubren sobre todo la capacitación y la asistencia técnica, los requerimientos de 
las pymes son más amplios en realidad, aunque principalmente se identificarían los financieros y apoyos integrales de asistencia técnica.

Frente a eso, algunas empresas han empezado a reforzar su responsabilidad social y, más especialmente, el aspecto relacionado con la sustentabilidad. Esta tendencia, que busca acercar las organizaciones a un nicho de mercado sensible a las cuestiones de ecología, desarrollando productos que cuidan del medio ambiente y una infinidad de acciones reales, comprobadas $y$ legítimas que dan un valor añadido a su negocio a través de la concientización ecológica. La sustentabilidad puede aportar herramientas para reorientar el comportamiento de las empresas con su entorno y redirigir el proceso de compra hacia un modelo sostenible.

La competitividad de las pymes para participar en el entorno globalizado, crear ambientes propicios para el desarrollo de esta y de sus agentes económicos permite la continuidad de las micro, pequeñas y medianas empresas, además de fomentar el desarrollo económico local y regional (Cabrero, 2019). Sin embargo, muchas empresas han decidido basar los procesos y decisiones sobre la sustentabilidad, sin integrarlo en una política real, transversal y sincera de Responsabilidad Social Corporativa.

Una de las problemáticas de las pymes es la inadecuada articulación del sistema económico, que favorece, a las grandes empresas son:

a. Políticas gubernamentales inadecuadas; b. borrupción administrativa de funcionarios del sector público; c. Falta de financiamiento o carestía del mismo., -Inapropiada infraestructura técnico-productiva; d. Carencia de recursos tecnológicos; e. casi nula aplicación de adecuados sistemas de planificación empresarial; f. Competencia desleal del comercio informal; g. Globalización y las prácticas desleales a nivel internacional; además de acuerdos y proyectos llevados a cabo, se necesitan recursos económicos que, a menudo, las Pymes no puede asumir. La empresa eco sostenible tiene que ser consciente de que su incursión en el mercado verde no es solo una herramienta, es todo un concepto que abarca cada una de las partes de su cadena productiva.

Otro de los tantos problemas en el desarrollo de la sustentabilidad es la falta de consecuencias de muchas empresas que asumen la etiqueta ecológica de la marca hacia afuera descuidando detalles de sus procesos productivos e incluso logísticos. Como en todo proceso productivo la eficiencia está en cómo reducir costos y obtener más por menos. En muchos casos una gestión ambientalmente sostenible de la empresa y sus productos puede muy bien reducir los costos a la vez que brinda un mejor producto y/o servicio a sus clientes.

Ciertamente para las empresas que aún no se hallan inmersas en este ámbito el lanzarse de lleno en esta nueva tendencia significa una fuerte y hasta chocante restructuración de sus procesos, pero el resultado puede ser de los más satisfactorios al involucrarles en un proceso de innovación constante, reposicionamiento de marca y apertura de nuevos mercados.

En forma concreta se puede decir que, de los problemas de las pymes, se basan fundamentalmente en el desconocimiento y la ausencia de normas para el desarrollo de un negocio sustentable.

Esta investigación se justifica 
teóricamente, porque estimula el desarrollo de futuras investigaciones relacionadas con herramientas gerenciales empresas sostenibles y el mercado ecológico. Asimismo, generará conocimiento sobre el campo gerencial y ambiental para lograr empresas sostenibles puesto que la gerencia ambiental aún está en los umbrales del comienzo. En cuanto a la justificación metodológica, se emplean técnicas y procedimientos de investigación científica, aunado al diseño de instrumentos que permitirá el logro de los objetivos previstos en la investigación, los cuales podrán ser aplicados con las debidas adaptaciones en futuras investigaciones que requiera del conocimiento en materia gerencial y ambiental.

\section{Desarrollo sustentable: aportaciones esenciales}

Numerosas definiciones se han elaborado a partir de la década de los cincuenta sobre este término, basadas, inicialmente, en elementos puramente económicos e incorporando, con el tiempo, otras nociones como niveles de pobreza, calidad de vida, sustentabilidad, distribución de riquezas, entre otros. Una de las definiciones más aceptadas ha sido la brindada por Todaro (2015) quien afirma que el desarrollo consiste en "un conjunto de transformaciones que hacen que el sistema social, ajustado a las necesidades básicas y a los deseos de los individuos, evolucione desde unas condiciones de vida que todos perciben como insatisfactorias hacia otra situación en que las condiciones materiales y espirituales de vida sean mejores".

En los últimos años, el tema del desarrollo sustentable y sus prácticas han sido de gran importancia, pero a su vez de grandes preocupaciones sobre el medio ambiente. Varios países incluido México están activos en las negociaciones internacionales y encuentros que tienen que ver con el impacto ambiental. De acuerdo con Saldivar (2015) la crisis de los paradigmas, bajo una perspectiva de crecimiento y desarrollo no contempla la sustentabilidad, sin embargo, ésta atañe en lo económico, en el desarrollo, en el ámbito social, así como en algunas otras áreas del conocimiento.

El desarrollo sustentable creado por la Comisión de Brundtland es un tema que intenta ir hacia otra vertiente con consecuencias en el consumo y la distribución, pese a ello aún existe un punto cuestionable: la definición de lo que es realmente sustentable. La precisión de "la sustentabilidad", encierra dos visiones contrapuestas, la visión económica y la visión científica. La visión económica está íntimamente relacionada con la concepción del desarrollo; la posibilidad de un desarrollo sustentable se relaciona con la creación de tecnologías viables que logren o hagan posible el crecimiento económico, sin las externalidades negativas que este produce o, por lo menos, minimizando las mismas.

De acuerdo a Jardón (2016) en el desarrollo sustentable existen dos aspectos a tomar en cuenta: 1. El aumento excesivo de energía requerido para la industrialización de los países reconocidos como postindustrializados, y la subsistencia como países desarrollados y 2. Los costos sociales y el "desarrollo" de la actividad económica de esas sociedades actuales donde se asegura el "crecimiento" no se desarrollan de la nada, dependen generalmente de un marco natural y de 
un deterioro ambiental.

Es importante conocer el concepto de desarrollo sustentable y como éste ha influenciado en gran medida "el crecimiento". Algunas disciplinas como la política, la sociología y la economía contribuyen para aclarar el significado y trascendencia de este conocimiento, pero, sobre todo, ayuda a comprender los obstáculos que este proceso conlleva.

Una definición de Desarrollo es la que proporciona Gómez (2009: 56) como el "conjunto de actuaciones, realizadas por actores públicos y privados, entre países de diferente nivel de renta con el propósito de promover el progreso económico y social de los países, que resulte sostenible". El concepto de desarrollo envuelve, conjuntos económicos y sociales, entre estos dos conjuntos es importante no dejar atrás la utilización de los recursos naturales y el orden del medio ambiente. En el mundo se pueden diferenciar los opuestos de las sociedades que muestran disparidad ante el bienestar y ante el desarrollo socioeconómico; es fundamental entender estos opuestos de las sociedades, profundizando en los conceptos basados en las grandes desigualdades. El primer razonamiento de diferenciación regional tiene que ver con el grado de desarrollo socioeconómico de los países y regiones, que poco tiene que ver con que se disponga de, más o de menos, recursos económicos.

Está relacionado con una multiplicidad de factores; hechos históricos, nivel cultural, nivel de desarrollo técnico de la sociedad, con el grado de eficacia en la cuestión económica, con la naturaleza de las relaciones espaciales internas o con el grado de control de las relaciones comerciales (Moraleda 2014).

Es decir, el desarrollo debe tener consigo el factor económico, pero está asociado a una trasformación de estructuras referidas al proceso estratégico, cuyo fin es propiciar un conjunto de transformaciones de la estructura económica y en la participación social, a través de cambios de fondo que corrijan desequilibrios fundamentales del apartado productivo y distributivo. Esto es, que además de asegurar su armonía con el medio ambiente, eran inherentes a un desarrollo con este calificativo, transformaciones gerenciales que permitiesen el cambio social gradual y un crecimiento económico auto sostenido.

El concepto de desarrollo sostenible refleja una creciente conciencia acerca de la contradicción que puede darse en el desarrollo, en que se entiende como crecimiento económico y mejoramiento del nivel material de nuestra vida; y las condiciones ecológicas y sociales, y de equidad para que ese desarrollo pueda perdurar en el tiempo. Esta conciencia de los costos humanos, naturales y medioambientales del desarrollo, y el progreso, han venido a modificar la actitud de despreocupación o justificación que al respecto, imperó durante mucho tiempo. La idea de un crecimiento económico sin límites y en pos del cual, todo podía sacrificarse, vino a ser reemplazada por una conciencia de esos límites y de la importancia de crear condiciones de largo plazo que hagan posible un bienestar para las actuales generaciones, que no se haga al precio de una amenaza o deterioro de las condiciones de vida futuras de la humanidad.

En términos más generales el desarrollo sostenible aborda tres áreas: económica, ambiental y social. En apoyo a esto, varios textos de las Naciones 
Unidas, incluyendo el Documento Final de la cumbre mundial en el 2005, se refieren a los tres componentes del desarrollo sostenible, que son el desarrollo económico, el desarrollo social y la protección del medio ambiente, como "pilares interdependientes que se refuerzan mutuamente" (Cumbre Mundial 2019).

\section{La gerencia y la sustentabilidad como instrumento de innovación}

La sustentabilidad puede ser un instrumento válido para aquellas situaciones en las que es posible mejorar una situación medioambiental determinada, mediante acciones que conduzcan al cambio de comportamiento de los individuos en el ámbito local, ya que, es más sencillo modificar comportamientos en segmentos reducidos que en la escala global.

Como se apreciara en la siguiente sección, a partir de la encuesta aplicada las actitudes y la conciencia ambiental no siempre se corresponden con los comportamientos que finalmente adoptan las personas $\mathrm{y}$, como consecuencia de ello, una mejora significativa en la conciencia ambiental no se traduce necesariamente en unos comportamientos más proambientales. Sin embargo, es más sencillo diseñar campañas de "concienciación" (utilizando esencialmente elementos de comunicación que poco o ningún resultado suelen tener), antes que tratar de desarrollar aquellas que tengan como objetivo la modificación de comportamientos.

Desde el nicho gerencial el líder como principal componente de la organización, piensa en la sustentabilidad como ventaja competitiva, al tomar en cuenta que el direccionar la empresa por la parte ambiental la está apalancando al éxito y sosteniéndola en la parte de la competitividad. Asumir este estadio, es abrirse a otros mercados específicamente, las empresas sostenibles son aquellas que están manejadas gerencialmente y cumplen con la disciplina de la organización para cristalizar sus objetivos, sean a corto mediano y largo plazo. Estas hacen esfuerzos sociales, tecnológicos y económicos para sembrar la sensibilidad ambiental a través de sus productos en nuevos consumidores.

La sostenibilidad empresarial descansa en la cultura ecológica, la cual está, interrelacionada con el desarrollo sustentable, consciente del ahorro de la energía, la justicia social, la equidad, la responsabilidad social corporativa y la ética. En América Latina está en su etapa inicial solo algunas empresas están dispuestas a llevar adelante este proceso aún falta por enseñar, sensibilizar al consumidor, es decir, que adquiera la cultura ecológica no solo para la compra de productos sino para ser agente multiplicador del cuidado ambiental y expresarlo a través de la conservación y defensa del entorno local en beneficio de todos los habitantes. El planeta está cada vez más envuelto en la vorágine de los cambios permanentes, que involucra la mayoría de los campos de estudio, la operatividad de las organizaciones y el pensamiento de la humanidad; así como, la apertura económica, la competencia internacional es cada vez mayor para los productos de la empresa, situación que ha desencadenado en la apertura de nuevos mercados, coyuntura que debería ser aprovechada por los gerentes que a su vez conlleva a la modernización de la empresa. Adicionalmente, incrementa la 
productividad, mejora la calidad de los productos y baja los costos operacionales con el propósito de aumentar la competitividad, se mejoraría el ingreso per cápita porque las empresas son el motor del desarrollo de la economía. La tendencia de los negocios es hacer fusiones 0 adquisiciones parciales, todas estas fusiones se caracterizan por disminuir costos de transporte y producción, por las economías de escala, aumentar la cobertura y la participación del mercado y facilitar el ingreso y apertura de nuevos mercados o para consolidarse y competir exitosamente, logrando dar mejor y oportuno servicio y reducir los costos al consumidor.

La globalización ha contribuido a la estandarización de criterios tecnológicos, calidad de productos, uso de materias primas, hasta la aplicación de nuevos enfoques gerenciales, tomando como punto de referencia el deterioro ambiental, como consecuencia del calentamiento global, situación que ha generado polémica en la esfera empresarial, a escala planetaria; pues hoy, la empresa tiene la necesidad de asumir nuevos modelos para sobrevivir en mercado competitivo, dado que, el proceso de producción, ocasiona alteraciones significativas en el medio ambiente. Actualmente, la empresa asume el desafío de generar riqueza, ser más competitiva, crear nuevos empleos, contribuir al desarrollo social del país emplear tecnologías más limpias, generar menos residuos, y tener equilibrio con el entorno. Vale decir que, esta posición no ha sido bien vista por muchos empresarios generando enfrentamiento y resistencia al cambio; pero esta contraposición empresasustentabilidad, ha sido superada en muchos países desarrollados, inclusive desde inicios del siglo pasado, aceptando los nuevos modelos de fabricación con el componente de la preservación ambiental. Situación que coincide con el desarrollo sustentable, donde su objetivo consiste en usar los recursos que necesite la comunidad, pero dejando en la mejor condición estos (recursos) para las futuras generaciones. Permitiendo un mejoramiento sostenido y equitativo de la calidad de vida de sus habitantes, asumiendo las políticas pertinentes a la sustentabilidad planetaria.

Durante las últimas décadas, la sustentabilidad ha experimentado un considerable desarrollo, paralelo a la propia evolución de las sociedades en las que se extiende su ámbito de actuación. En la medida en que las necesidades y la calidad de vida de las personas se han visto incrementadas por el impulso de los agentes económicos y sociales, las empresas se han adaptado a estas nuevas tendencias mediante la utilización de mejores y modernas técnicas sustentables, capaces de satisfacer más favorablemente dichas necesidades y contribuyendo con ello a un mayor bienestar general de la población. Se conciben nuevas formas de entender los mercados y a los consumidores, se utilizan nuevos instrumentos $y$ tecnologías para obtener información, se buscan nuevos métodos para desarrollar las relaciones de intercambio con los consumidores y se potencian nuevas formas de comunicación con ellos.

La conciencia ecológica y él consumo ético comienzan a jugar un rol protagónico en las estrategias de comunicación de las empresas. Las que no se adecuen a un tipo de negocio sustentable y socialmente responsables, a la larga, serán castigadas por los consumidores, ya que estos comienzan a exigir a las marcas que adopten una postura solidaria, tanto 
con el medio ambiente como con las sociedades.

La capacidad para competir en los mercados de bienes y servicios que imperan en la industria y la región por medio de los mejores métodos de producción y de organización genera ventajas competitivas para el desarrollo y éxito. En México los programas de apoyo para las pymes cubren sobre todo la capacitación y la asistencia técnica Mckenzie y Mohr (2015), los requerimientos de las pymes son más amplios en realidad, aunque principalmente se identifican los financieros y apoyos integrales de asistencia técnica.

Frente a eso, algunas empresas han empezado a reforzar su responsabilidad social $y$, más especialmente, el aspecto relacionado con la sustentabilidad. Esta tendencia, que busca acercar las organizaciones a un nicho de mercado sensible a las cuestiones de ecología, desarrollando productos que cuidan del medio ambiente y una infinidad de acciones reales, comprobadas y legítimas que dan un valor añadido a su negocio a través de la concientización ecológica. El gerente puede aportar herramientas para reorientar el comportamiento de las empresas con su entorno y redirigir el proceso de compra hacia un modelo sostenible.

En este sentido, es necesario que quede claro que no se trata de enfrentar a los objetivos económicos de cada variable de marketing con los objetivos sustentables, se trata de buscar la consecución de ambos a la vez. Adoptar la filosofía de la sustentabilidad exige que el respeto por el medio ambiente impregne todo el comportamiento de la organización, no solamente los aspectos comerciales. Un producto no debería ser comercializado como ecológico centrándose únicamente en el producto en sí, mientras ignora las implicaciones ecológicas de la fabricación y de la compañía en su conjunto (Rodríguez 2016).

Tomando en cuenta las definiciones de los diversos autores expertos en la materia, se puede comentar que la sustentabilidad es un conjunto total de actividades para crear, comunicar y entregar valor a los clientes y la sociedad en general, el cual se deriva del intercambio entre las relaciones sociales, a fin de lograr los objetivos de la organización y satisfacer las necesidades de los mercados a través del desarrollo y estudio de variables comprendidas dentro de dicho marco.

Por su parte los autores, Rodriguez y Ponce (2016) consideran que la responsabilidad social de la empresa es incrementar sus beneficios. Destacan que la empresa sostenible ha de actuar de forma coherente con los principios del desarrollo sostenible; considerando que el valor central de la empresa sostenible no es crecimiento económico sino el desarrollo sostenible, pues, el objetivo es triple: crear valor económico, medio ambiental y social. (Triple botton line).

Partiendo del principio que el capital humano es el nervio que opera diversas empresas y dentro de este, el gerente, es la persona que lleva la función de administrar cualquier nivel jerárquico de la organización, para concretar las metas. De manera que, el gerente es el ente que maneja la empresa, que está pendiente de todo lo que amerita para la funcionalidad de la organización, para ejecutar las actividades y funciones inherentes $y$ adecuadas para el propósito esperado de la mencionada organización. Vale 
decir, que las funciones del gerente son: planificación, organización, dirección, control y en conjunto se conoce como proceso administrativo.

En correspondencia con lo anterior, tal como lo expresa García (2017) considera que el gerente mantiene el equilibrio de la empresa, que dirige, incrementa la producción, plantea soluciones, brinda protección, es considerado el cerebro interactivo de la organización.

\section{Sustentabilidad en las pymes de Jalisco}

Se tomó una muestra aleatoria de los consumidores en la zona metropolitana de Jalisco. El instrumento utilizado permitió realizar un diagnóstico sobre el conocimiento que se tiene, acerca de la sustentabilidad y su estrategia en las empresas, las características y condiciones de las pequeñas y medianas empresas que se encuentran dentro de la rama de la sustentabilidad en el estado de Jalisco.

Se utilizó la versión de Sánchez et al, (2008), en la elaboración del cuestionario tomando en cuenta las diversas definiciones sobre sustentabilidad, con un diseño de 12 Ítems, se siguió de dos procedimientos: 1. se analizó la variación de la encuesta para la eliminación de ciertos ítems para mejorar su fiabilidad y 2 . se realizó un análisis factorial exploratorio para mejorar su dimensionalidad.

Las respuestas de los encuestados fueron evaluadas con una escala de 1 al 5 donde evaluaban con $1=$ Totalmente en desacuerdo, 2= Desacuerdo, 3= Neutral, 4= De acuerdo, 5= Totalmente de acuerdo, contando con 25 preguntas todas ellas en una escala de Richter. Se empleó un análisis de varianza llamado
(ANOVA) para verificar los cruces de variables, su correlación y su nivel de significancia con los resultados obtenidos del cuestionario. Se utilizó el programa Lime Survey, como software, este nos importa los datos al siguiente software estadístico llamado SPSS, donde nos permitió, entre otras funcionalidades, la gestión de datos, el análisis estadístico, el trazado de anovas, gráficos y simulaciones.

Se realizó una encuesta con una muestra de 400 consumidores de los productos sustentables donde las pymes han empleado dicha estrategia, el propósito fue, analizar si la sustentabilidad aplicada en la pymes funciona como estrategia que proporciona competitividad. Se envió el cuestionario vía enlace por medio del programa Lime Survey, a la población objetivo donde se obtuvo una respuesta de 196 personas del género femenino, y 204 personas del género masculino quienes consumen productos con dicha estrategia, el porcentaje por géneros, es del 49 por ciento del género femenino y 51 por ciento por el género masculino.

En la escala de Fisher, al hacer el cruce en donde al encuestado se le pregunta: ¿Si la sustentabilidad se utiliza como estrategia competitiva y esta crea un diferencial en la marca? Da una escala de 79.549 y un nivel de significancia de .000 altamente confiable, además de aparecer en el inter-grupo, lo que nos da como respuesta que la modificación de crear el diferencial de la marca por medio de la estrategia debe realizarse dentro de la organización.

Por otra parte al hacer el cruce donde se les pregunta a los encuestados ¿Si la sustentabilidad se utiliza como estrategia y esta logra conectarse con la conciencia de las personas, compartiendo con ellas sus principios 
o ideales? Esta da como resultado un nivel de significancia de .000 con un escala de Fisher de 17.630, indicando en la media cuadrática que se trata de una modificación o estrategia que la compañía debe de realizar en el intragrupo, es decir dentro de la organización.

Se les pregunta ¿Si una empresa que no gestiona la sustentabilidad puede ser castigada por los consumidores? Muestra que en la escala de Fisher es de 13.711 mientras que el nivel de significancia es de 0.000 , por otra parte en la media cuadrática casi por partes iguales nos arroja las cifra de 1.902 en el intergrupo y 1.387 en el intragrupo donde nos indica que las estrategias deben implementarse en ambas áreas, es decir dentro y fuera de la organización, la pyme debe gestionar la sustentabilidad dentro de sus procesos, pero es indispensable también que esta, los comunique hacía el exterior por medio de sus productos. Los resultados que contempla y debería asumir la pyme en cuestión de una estrategia de sustentabilidad están basados en seis variables que la conforman y estas son las siguientes: 1 . consideraciones ecológicas, 2. eco etiquetado, 3. envase ecológico, 4. envase retornable, 5.consideraciones ecológicas en sus procesos y distribución, 6.proporcionar información ecológica al mercado. Respecto a los resultados y hallazgos se exponen cinco consideraciones operacionales para la pyme, estas son: 1. minimización en el uso de materiales y recursos no renovables, 2 . sustitución de materiales no ecológicos, 3 . uso de los criterios medioambientales para el proceso de fabricación, 4. optimización $y$ uso racional de los materiales y procesos, 5. Implementación de los nuevos procesos tecnológicos para ahorrar energía y agua.

Estas acciones incrementan la relación entre la cantidad de productos obtenida por un sistema productivo y los recursos utilizados para obtener dicha producción, con un incremento de la productividad como indicador de eficiencia. El incremento de la productividad les dará beneficios corporativos a las pymes a corto y largo plazo. En conjunto el incremento de la productividad que a su vez genera beneficios tanto económicos como ambientales, a corto y largo plazo, le dará un valor agregado a la mejora de imagen en la pyme, incrementando la competitividad, haciendo así un ciclo de beneficios que fomenta la competitividad en la sustentabilidad como estrategia.

Asimismo, se incluyen las políticas, procedimientos y controles formales que guían las actividades y relaciones de la gerencia con la pyme, empleando cuatro acciones dentro de la variable de estructuras que son: diseño de objetivos medioambientales mesurables, formalización de procesos para identificar requisitos legales, desarrollo de programas para alcanzar los objetivos medioambientales y la definición clara de responsabilidades para implementar las estrategias de sustentabilidad.

\section{Conclusiones}

La sustentabilidad y el fenómeno organizativo de la implementación de esta como estrategia en las pymes, en la presente investigación realizada, permitió conocer la identidad teórica y empírica, que recogío las principales acciones de la sustentabilidad, la cual, además de tener un nivel aceptable de fiabilidad, también mostro su unidimensionalidad a través de un análisis factorial comprobatorio. 
Por ello la encuesta desarrollada se podría considerar un aporte al conocimiento de la sustentabilidad como estrategia competitiva en las pymes, la investigación además constituye un aporte, tanto en cuanto valida dos modelos que integra sus variables individuales y organizativas.

Se determina que las pymes particularmente hablando del estado de Jalisco son propensas a la falta de estrategias sustentables, que no permiten enfrentar los movimientos externos y de competitividad, por la ausencia de una estructura legal, corporativa y de políticas de fomento hacía la sustentabilidad.

Igualmente, las empresas deben conocer los elementos del entorno que pueden afectar lo capacidad de toma de decisiones, así como identificar la influencia que estos pueden ejercer en el comportamiento estratégico de la firma. Ello concuerda con el hecho de que se pueden concebir a las empresas como entidades que basan su heterogeneidad, y por consiguiente su competitividad, en el conocimiento de la sustentabilidad. Así mismo la sustentabilidad debe ser considerado como una capacidad que puede generar una ventaja competitiva sostenible para la pyme.

La implementación de los resultados sugiere que la estrategia de la sustentabilidad medioambiental se encuentra de manera deficiente en las organizaciones, donde se requiere una mayor contribución por parte de los académicos, para proponer herramientas que puedan alcanzar y/o extender el conocimiento teórico- práctico de la sustentabilidad como estrategia.

La muestra de población de 400 personas consumidoras de dichos productos, determina que las variables administrativas desarrolladas por las pymes para implementar la sustentabilidad como estrategia son aún deficientes. Estos resultados son coherentes con el estado de resultados que nos da al hacer el cruce de nuestras variables, además la literatura indica que la influencia de los consumidores y el sondeo de los mismos, es uno de los factores más importantes para el éxito en la formulación e implementación de las estrategias competitivas (Urbano 2018), dado que sus percepciones influyen en su conducta con el entorno y con los diferentes grupos de presión medioambiental (Nickels 2017) y ejercen un poderoso impacto en la formación de la cultura medioambiental de la empresa (Valdés 2016).

La organización puede crear valor si es que usan los sondeos de los consumidores para administrar las variables organizativas mejor que la competencia. Igualmente, las pymes deben conocer los elementos del entorno que pueden afectar lo capacidad de toma de decisiones, así como identificar la influencia que estos pueden ejercer en el comportamiento estratégico (Hunt 2009). Ello concuerda con el hecho de que se pueden concebir a las empresas como entidades que basan su heterogeneidad, y por consiguiente su competitividad, en el conocimiento de la sustentabilidad. Así mismo la sustentabilidad debe ser considerada como una capacidad que puede generar una ventaja competitiva sostenible para la pyme.

\section{Referencias Bibliográficas}

Agarwal, Anil; Narain, Sunita (2001), Global Warning in an Unequal World. A Case of Environmental Colonialism. Centre for Science and Environment.

Carrioza, Julio. (2012), La Politica Ambiental en Colombia: Desarrollo Sos- 
tenible y Democratización. Colombia: Artes Colombia.

CGR. (2005), Informe de auditoria especial al proyecto PNUD - GEF COL /99/G31/A/1G/99 Cambio Climático. Bogota: IDEAM, FASE II.

CMCC. (08 de 11 de 2014), International Insitute for Sustainable Development. Obtenido de International Insitute for Sustainable Development: http://www.unfccc.int

CONFERENCIA DE PARTES.(10 de 02 de 2015), Conferencia de las Partes. Obtenido de Conferencia de las Partes: http://finanzascarbono.org/financiamiento-climatico/canales-multilaterales-de-financiamiento/cmnucc/ cop/

Escobar, Arturo (2004), El desarrollo Sostenible: diálogo de discursos. Foro No. 23.

Fermann, G. (2017), Political context of climate change. Oslo: University Press.

FMAM. (2011). Global Environment Facility. BID. Obtenido de FMAM: http://www.iadb.org/es/ temas/medio-ambiente/fondo-para-el-medio-ambiente-mundial/quees-el-fondo-para-el-medio-ambientemundial-fmam, 1701.html

Grubb, Michael; Sebenius, James; Magalhaes, Antonio; \& Subak, Susan (2012), Sharing the burden.Confronting Climate Change. Stockholm Environment Institute. Cambridge University.

Herzog, Tim (2009), Word greenhouse gas emissions. World Resources Institute.

Protocolo de Kyoto, (01 de Febrero de 2019), Instituto Nacional de Ecología y Cambio Climático. Obtenido de Instituto Nacional de Ecología y Cambio Climático: http://www.inecc.gob.mx/ cpcc-agenda-int/650-cpcc-texto-kioto

Kyoto, Protocol (15 enero de 2019), Finanzas Carbono. Obtenido de Finanzas Carbono: http://finanzascarbono. org/financiamiento-climatico/canales-multilaterales-de-financiamiento/ protocolo-kioto/

Martín, Jesus Ramos (2012), De Kyoto a Marrakech: Historia de una flexibilizacion anunciada. Universidad Autonoma de Barcelona.

Nations, United (03 de 01 de 2015), Kyoto Protocol to the United Nations Framework Convention on Climate Change. Obtenido de Kyoto Protocol to the United Nations Framework Convention on Climate Change: http://unfccc.int/essential background/kyoto_protocolo/items/1678. php

Parikh, J (2015), Join Implementation and North -South Cooperation for Climate Change. International Environmental Affairs, Vol 7 (1): 22-41.

Paterson, Matthew (2003), The Politics of Climate Change after UNCED. Environmental Politics, Vol. 2 (4).

United Nations Framework Convetion On Climate Change (1998), The Buenos Aires Plan of Action. Report of Conference of the Parties on its Fourth Session, held at Buenos Aires from 2 to 14 november 1998. Buenos Aires.

United Nations Framework Convetion On Climate Change (5 de 12 de 2019), Activities Implemented Jointly. Obtenido de Activities Implemented Jointly: http://www.unfccc.int

United Nations Framework Convetion On Climate Change (08 de 02 de 2019), Kyoto Protocol to the Nations Framework Convention on Climate Change. Obtenido de Kyoto Protocol to the Nations Framework Convention on Climate Change: http://www. 
Sustentabilidad como estrategia competitiva en la gerencia de las pymes en México Ortiz Palafox, Karla Haydee

unfccc.int

United Nations Framework Convetion

On Climate Change (2019), Kyo-

to Protocol to the United Nations

Framework Convention on Climate
Change. FCCC disponible en http:// www.unfccc.int.

- Esta obra está bajo una licencia de Creative Commons Reconocimiento-NoComercialCompartirlgual 3.0 Unported.

http://creativecommons.org/licenses/by-nc-sa/3.0/deed.es_ES 